\title{
Perceived Stress and Emotional Eating in Adolescence: Mediation Through Negative-Focused Cognitive Emotion Regulation and Reward Sensitivity
}

\author{
Jiaai Huang \\ Wuhan Sports University \\ Yuanjie Wang \\ Wuhan Sports University \\ $\mathrm{Na} \mathrm{Ye}$ \\ Wuhan Sports University \\ Xia Xu ( $\square$ xuxia@whsu.edu.cn ) \\ Wuhan Sports University https://orcid.org/0000-0002-1285-819X
}

\section{Research article}

Keywords: Emotional eating, Adolescent, Stress, Cognitive emotion regulation, Reward sensitivity

Posted Date: December 28th, 2020

DOl: https://doi.org/10.21203/rs.3.rs-133643/v1

License: (c) (1) This work is licensed under a Creative Commons Attribution 4.0 International License.

Read Full License 
1 Perceived Stress and Emotional Eating in Adolescence: Mediation through

2 Negative-focused Cognitive Emotion Regulation and Reward Sensitivity

3

4 Jiaai Huang ${ }^{1}$, Yuanjie Wang ${ }^{1}, \mathrm{Na} \mathrm{Ye}^{2,3}$, and $\mathrm{Xia} \mathrm{Xu}^{2,3^{*}}$

5

$6 \quad{ }^{1}$ Graduate School, Wuhan Sports University, Wuhan, China

$7 \quad{ }^{2}$ Hubei Key Laboratory of Sport Training and Monitoring, College of Health Science,

8 Wuhan Sports University, Wuhan 430079, China

$9{ }^{3}$ Scientific Sports and Health Promotion Hubei Collaborative Innovation Center,

10 Wuhan 430079, China

11

$12{ }^{*}$ Correspondence:

13 Xia Xu, Hubei Key Laboratory of Sport Training and Monitoring, College of Health

14 Science, Wuhan Sports University, 461 Luoyu Road, Wuhan City, Hubei Province 15 430079, China.

16 Email: xuxia@whsu.edu.cn 


\section{Abstract}

Background: There have been ambiguous findings on the empirical relationship between perceived stress and emotional eating. The mediating roles of negative-focused cognitive emotion regulation and reward sensitivity of these relationships, particularly for adolescents, are often overlooked. The objective of this study was to assess the association between perceived stress, negative-focused cognitive emotion regulation, reward sensitivity, and emotional eating in a sample of Chinese adolescents.

Methods: In this cross-sectional study, 562 adolescents were selected and evaluated based on perceived stress, negative-focused cognitive emotion regulation, reward sensitivity, and emotional eating. Model tests were conducted using serial multiple mediation analyses, controlling for sex, age, household income, and body mass index.

Results: Results showed that perceived stress directly affected adolescents' emotional eating. In addition, perceived stress indirectly affected emotional eating through negative-focused cognitive emotion regulation and reward sensitivity.

Conclusions: Findings support the hypothesis that perceived stress increases negative-focused cognitive emotion regulation, which increases reward sensitivity and thus increases emotional eating. Implications of these findings for preventive and therapeutic intervention are discussed, and future research recommendations are provided.

Keywords: Emotional eating, Adolescent, Stress, Cognitive emotion regulation, Reward sensitivity 


\section{Background}

Emotional eating (EE), defined as eating in response to negative emotions [1], has been explained as a coping mechanism during moments of stress, whereby people try to 'eat their troubles away' with highly palatable foods which are rich in sugar, fat and calories [2]. However, emotional eating will not solve the stress origins, the stressor will remain and cause psychological discomfort $[3,4]$. Thus, emotional eating creates a vicious circle. Emotional eating may lead to overweight or obesity over time and even evolve into an eating disorder (e.g., binge eating disorder), prediabetes and diabetes $[2,5]$. These negative consequences emphasize the importance of obtaining further insight into emotional eating.

Stress arises when the demands being made on individuals or threats to their well-being exceed their available coping resources [6]. A meta-analysis concluded that stress was negatively associated with healthy eating behaviours in children aged 13-18 years old [7]. In several studies, children's and adolescents' perceived stress have been associated with high scores on emotional eating $[8,9]$. Vandewalle et al. [10] also found that daily stress is positively associated with daily fluctuations in scores on emotional eating. However, others observed exactly the opposite, they found individuals eat less or report no change during stressful situations compared to non-stressful situations [11-13]. According to the models of stress-induced eating [14, 15], stress induces eating through physiological and psychological processes. Thus, the perspective that some factors mediated the effect of stress on emotional eating 
may help to account for inconsistent empirical findings.

Cognitive emotion regulation (CER), or cognitive coping, generally refers to the cognitive way of managing the intake of emotionally arousing information during or after the experience of threatening or stressful events. Stress can affect cognitive emotion regulation [16], thus rendering individuals even more susceptible to emotional processes that can trigger unhealthy eating. Based on the long-term effects on affect, behaviour, and psychopathology, researchers suggest a classification in adaptive strategies and maladaptive strategies [17, 18]. CER strategies influence eating behaviour $[19,20]$. Researchers hypothesize that individuals having difficulty regulating their emotions often turn to substances such as alcohol or food $[2,21]$. Studies in children and adolescents have revealed an association between maladaptive strategies and emotional eating [22-25]. This association is stronger when maladaptive strategies are used to deal with the stressor in comparison with the use of adaptive strategies [26]. These results suggest that a lack of adaptive strategies is not necessarily linked to emotional eating, but rather to the use of maladaptive strategies, whereas CER seems to mediate the stress-eating relationship [2, 19, 27]. The research on CER offers an interesting perspective on the problem of the relationship between stress and emotional eating.

Reward sensitivity (RS) is a psychobiological personality trait, referring to an individual's ability to experience pleasure or reward on exposure to appetitive stimuli [28]. RS increases from childhood to adulthood with a peak in adolescence, RS might thus play a substantive role in explaining adolescents' eating behaviours [29, 30]. For 
example, De Cock et al. [31] found that RS was associated with snack and sugar- ${ }^{-}$sweetened beverage consumption in adolescents aged 14-16 years, especially in girls. Stress makes people susceptible to substances that, via reward processing, motivate individuals to overconsume them [32]. Previous findings provide preliminary evidence that stress-reactive individuals show diminished RS under stress [33]. A review concluded that acute and chronic stress have dissociable effects on RS [34]. Specifically, acute stress is linked to an increase in incentive motivation and active coping behaviours, but chronic stress abolishes these behaviours and makes individuals become blunted with repeated stressors, reflected in a change in behaviour from active coping to learned helplessness and reduced RS. In addition, research on the determinants of emotional eating has also suggested the influence of individual traits such as sensitivity to food and reward [2]. Researchers reported that RS was related to emotional eating in adolescents [31] and adults [35]. Moreover, increased RS has been found to be associated with vulnerability to develop binge eating behaviour [36]. Several studies have revealed a positive, negative or no association between coping strategies and RS [37-40]. The research on CER and RS will enrich our understanding of stress-eating links.

Adolescence is a critical developmental period characterized by socioemotional and academic challenges where they are particularly vulnerable to detrimental consequences of stress. Simultaneously, they are gaining autonomy including with respect to food choices from parents. The purpose of this study to verify the mediating roles of negative-focused cognitive emotion regulation and reward sensitivity in the 
111 synergic effect of the relationship between perceived stress and emotional eating. We

112 hypothesized that negative-focused cognitive emotion regulation and reward

113 sensitivity act as mediators between perceived stress and emotional eating among

114 adolescents. Although these potentially associated constructs have been examined

115 separately in the past, by incorporating both, this study will also allow for

116 examination of the interrelationships between these variables. Examination of this

117 issue may provide empirical foundations for prevention and intervention strategies

118 tailored to concerns of adolescents.

\section{Methods}

\section{Sampling and data collection}

This was a cross-sectional study, conducted between October and November 2019, which enrolled 578 young adolescents aged between 12 and 15 years using a sample from Wuhan City, China (Central China). A total of five junior high schools were contacted randomly, of which two agreed to participate. We obtain informed consent from students and their legal guardians by sending letters to legal guardians through their schools. They had the choice to agree or to decline to participate in the study. Students who declined to fill the survey were excluded from this study. Questionnaires were self-administered and with no identification markers included in the data collection to ensure participant anonymity. Students needed approximately 20

$131-30 \mathrm{~min}$ to be complete the data collection. Participants were excluded for providing implausible responses (e.g., choose the last of all the options) to any of questionnaires 
( $n=9$ ), or failing to answer any of the following variables: sex, age, height, and household income $(\mathrm{n}=7)$. Self-reported height and weight were used to calculate each participant's BMI $\left(\mathrm{kg} / \mathrm{m}^{2}\right)$. The final sample was comprised of 562 adolescents $\left(12-15\right.$ years; mean age $=13.23$ years, $\mathrm{SD}=0.87$; mean $\mathrm{BMI}=19.00 \mathrm{~kg} / \mathrm{m}^{2}, \mathrm{SD}=$ $2.72 ; 51.60 \%$ males).

\section{Measures}

\section{Perceived Stress Scale (PSS)}

Perceived stress (PS) was measured using the 10-item PSS, a validated measure among adolescents [41]. The participants were asked how unpredictable, uncontrollable, and overloaded were the situations in the last month using a 5-point Likert scale from 0 (never) to 4 (very often). Total scores ranged from 0 to 40 , and participants with higher scores had higher perceived stress levels. Internal consistency for the full 10-item scale was good in our sample (Cronbach's alpha $=0.90)$.

\section{The Dutch Eating Behaviour Questionnaire - child version (DEBQ - C)}

DEBQ - $\mathrm{C}$ was used as a measure of adolescents' emotional eating (i.e., desire to eat under different negative emotional states, such as irritated, depressed, lonely, frightened or disappointed). This is a unidimensional instrument with 13 items answered on a 5-point Likert scale from 1 (never) to 5 (very often), with higher scores reflecting more emotional eating. Studies have indicated the usefulness of the DEBQ -

$\mathrm{C}$ in children and adolescents aged between 7 and 17 years [2]. In our study, Cronbach's alpha was 0.96 .

\section{The Cognitive Emotion Regulation Questionnaire (CERQ)}


Negative-focused cognitive emotion regulation (NFCER) was measured using the 4 maladaptive strategy subscales (self-blame, rumination, catastrophizing, and blaming others, 16-items) of the CERQ, which is a self-report assessment of an individual's cognitive emotion regulation strategies for stressful events or situation in general. The maladaptive strategy subscales are interpreted as "negatively focused cognitive emotion regulation". Self-blame refers to thoughts of blaming yourself for what you have experienced. Rumination or focus on thought refers to thinking about the feelings and thoughts associated with the negative event. Catastrophizing refers to thoughts of explicitly emphasizing the terror of an experience. Blaming others refers to thoughts of putting the blame of what you have experienced on others. The CERQ can be administered to people aged 12 years and older [18]. Responders indicate their degree of agreement with a 5-point Likert scale ranging from 1 [(almost) never] to 5 [(almost) always]. Higher total scores denote greater use of negative-focused cognitive strategies. Internal consistency in our study was good (0.89).

\section{The Behavioural Activation Scales (BAS)}

Reward sensitivity was assessed with the BAS. These scales consisted of three subscales: reward responsiveness (5 items), drive (4 items) and fun seeking (4 items), namely the anticipation of and the positive response toward a reward, the persistence to obtain goals, and the willingness to seek out and spontaneously approach potentially rewarding experiences. All items are to be answered on a 4-point scale from 1 (totally disagree) to 4 (totally agree). Higher total scores indicate higher levels of the reward sensitivity. The scale is a valid measurement of RS in children and 
adolescents [28]. In our sample the Cronbach's alpha was assessed as good for BAS (0.85).

\section{Statistical analysis}

Analyses were performed using SPSS version 25.0. Descriptive analysis, independent-samples T-test and one-way analysis of variance (ANOVA) were used to describe and compare the demographic data (sex, age, household income, and BMI) and questionnaires' scores. To yield standardized coefficients, all variables were converted to z-scores prior to analysis. Pearson's correlations were used to examine associations between study variables. To test the significance of the multiple-mediator model in this study, we adopted the model 6 of PROCESS v2.16 for SPSS provided by Hayes [42]. This approach was based on ordinary least-squares regression and the bootstrap method. Compared with the causal steps approach or the Sobel test, Hayes considered bootstrapping as the most powerful approach of assessment of indirect effects, as it was free from assumptions regarding the shape of the sampling distribution of the indirect effect and also had better control on type I errors. Hayes recommended 10000 bootstrap bias-corrected 95\% confidence intervals $(\mathrm{CI})$ to be used for mediation analyses in the test from the Serial-Multiple Mediation Model 6, and if they did not contain zero, they were considered significant [43]. PROCESS macro was performed using one independent variable (perceived stress), two mediators (negative-focused cognitive emotion regulation and reward sensitivity), and one dependent variable (emotional eating). Furthermore, both the direct and the total effect were tested. The serial mediation process estimated three specific indirect 
199 effects from perceived stress to emotional eating: (a) the indirect path via 200 negative-focused cognitive emotion regulation (PS $\rightarrow$ NFCER $\rightarrow$ EE); (b) the indirect 201 path via reward sensitivity (PS $\rightarrow$ RS $\rightarrow$ EE); and (c) the indirect path via 202 negative-focused cognitive emotion regulation to reward sensitivity (PS $\rightarrow$ NFCER $203 \rightarrow$ RS $\rightarrow$ EE). Finally, sex, age, household income, and BMI were treated as 204 covariates, and all covariates were controlled for at the level of both the mediator and 205 the outcome.

206 All variables were measured by self-report questionnaires so there might be the 207 problem of common method variance. The Harman single factor test showed that 9 208 factors were extracted, with the largest one accounting for $24.047 \%$ of the total 209 variance, indicating that common method variance is not likely to influence our results [44].

\section{Results}

\section{Preliminary Analyses}

214 Tables 1 categorical participant demographics, respectively, presented by gender 215 group. As shown in Tables 1, significant mean group (male vs. female) differences emerged for perceived stress, negative-focused cognitive emotion regulation and

217 emotional eating. One-way analysis of variance showed there were no significant 218 differences in household income related to age $(\mathrm{F}=1.473, \mathrm{p}=0.221)$, perceived stress $219(\mathrm{~F}=1.568, \mathrm{p}=0.196)$, negative-focused cognitive emotion regulation $(\mathrm{F}=1.023, \mathrm{p}=$ $0.382)$, reward sensitivity $(\mathrm{F}=0.854, \mathrm{p}=0.465)$, and emotional eating $(\mathrm{F}=0.980, \mathrm{p}=$ 
223 indicated that $<30000 \mathrm{CNY}$ household income participants had lower BMI (mean

$\left.224 \mathrm{BMI}=17.03 \mathrm{~kg} / \mathrm{m}^{2}, \mathrm{SD}=1.61\right)$ compared to other participants, and $\geq 100000 \mathrm{CNY}$

225 household income participants had lower BMI (mean BMI $=18.17 \mathrm{~kg} / \mathrm{m}^{2}, \mathrm{SD}=2.36$ )

226 compared to 30000-49999 CNY and 50000-99999 CNY household income

227 participants, whereas no difference was found between 30000-49999 CNY (mean

$\left.228 \mathrm{BMI}=20.02 \mathrm{~kg} / \mathrm{m}^{2}, \mathrm{SD}=3.40\right)$ and 50000-99999 $\mathrm{CNY}\left(\right.$ mean BMI $=19.93 \mathrm{~kg} / \mathrm{m}^{2}$,

$229 \mathrm{SD}=2.43$ ) household income participants.

230 Table 1 Demographic Characteristics and Study Variables for Study

231 Participants

\begin{tabular}{|c|c|c|c|c|c|c|c|c|}
\hline \multirow[b]{2}{*}{ Characteristics } & \multicolumn{2}{|c|}{$\begin{array}{c}\text { Male } \\
(\mathrm{n}=290)\end{array}$} & \multicolumn{2}{|c|}{$\begin{array}{l}\text { Female } \\
(\mathrm{n}=272)\end{array}$} & \multirow[b]{2}{*}{$\mathrm{t}$} & \multirow[b]{2}{*}{$\mathrm{p}$} & \multicolumn{2}{|c|}{$\begin{array}{c}\text { Total } \\
(\mathrm{n}=562)\end{array}$} \\
\hline & M & SD & M & SD & & & M & SD \\
\hline Age, years & 13.280 & 0.877 & 13.190 & 0.858 & 1.253 & 0.211 & 13.230 & 0.868 \\
\hline BMI, kg/m² & 19.101 & 2.936 & 18.885 & 2.467 & 0.943 & 0.346 & 18.996 & 2.719 \\
\hline Perceived stress & 7.990 & 7.556 & 12.670 & 8.659 & -6.848 & 0.000 & 10.250 & 8.434 \\
\hline $\begin{array}{l}\text { Negative-focused cognitive } \\
\text { emotion regulation }\end{array}$ & 33.910 & 11.251 & 36.590 & 10.773 & -2.878 & 0.004 & 35.210 & 11.094 \\
\hline Reward sensitivity & 36.260 & 6.872 & 37.050 & 7.168 & -1.338 & 0.181 & 36.640 & 7.022 \\
\hline \multirow[t]{3}{*}{ Emotional eating } & 23.070 & 12.297 & 26.720 & 13.271 & -3.386 & 0.001 & 24.840 & 12.897 \\
\hline & \multicolumn{2}{|c|}{$\begin{array}{c}\text { Male } \\
(\mathrm{n}=290)\end{array}$} & \multicolumn{2}{|c|}{$\begin{array}{c}\text { Female } \\
(\mathrm{n}=272)\end{array}$} & & & \multicolumn{2}{|c|}{$\begin{array}{c}\text { Total } \\
(\mathrm{n}=562)\end{array}$} \\
\hline & $\mathrm{n}$ & $\%$ & $\mathrm{n}$ & $\%$ & & & $\mathrm{n}$ & $\%$ \\
\hline \multicolumn{9}{|l|}{ Household income } \\
\hline$<30000 \mathrm{CNY}$ & 32 & 11.0 & 27 & 9.9 & & & 59 & 10.5 \\
\hline 30000-49999 CNY & 44 & 15.2 & 41 & 15.1 & & & 85 & 15.1 \\
\hline 50000-99999 CNY & 113 & 39.0 & 99 & 36.4 & & & 212 & 37.7 \\
\hline$\geq 100000 \mathrm{CNY}$ & 101 & 34.8 & 105 & 38.6 & & & 206 & 36.7 \\
\hline
\end{tabular}


234 Pearson correlation coefficients showed that age was negatively related to perceived

235 stress $(\mathrm{r}=-0.107, \mathrm{p}=0.011)$ and emotional eating $(\mathrm{r}=-0.111, \mathrm{p}=0.008)$, and

236 positively related to reward sensitivity $(r=0.122, p=0.004)$, yet no significant

237 correlation between age and negative-focused cognitive emotion regulation $(r=0.011$,

$238 \mathrm{p}=0.797)$, and BMI $(\mathrm{r}=0.070, \mathrm{p}=0.098)$. Also, there were no significant correlation

239 between BMI and perceived stress $(r=0.035, p=0.403)$, emotional eating $(r=0.027$,

$240 \mathrm{p}=0.529)$, negative-focused cognitive emotion regulation $(r=0.001, p=0.974)$ and

241 reward sensitivity $(\mathrm{r}=0.072, \mathrm{p}=0.087)$.

242 The correlations between the four study variables are shown in Table 2. Perceived

243 stress was positively related to emotional eating, negative-focused cognitive emotion

244 regulation and reward sensitivity. These bivariate correlations support the following

245 mediation analyses. In addition, there were significant correlation between emotional

246 eating and 4 maladaptive strategies of cognitive emotion regulation (self-blame, $r=$

2470.176 ; rumination, $r=0.180$; catastrophizing, $r=0.221$; blaming others, $r=0.206 ; p=$

$248 \quad 0.000)$.

Table 2 Correlations between perceived stress, negative-focused cognitive emotion regulation, reward sensitivity, and emotional eating

\begin{tabular}{|c|c|c|c|c|}
\hline Variable & 1 & 2 & 3 & 4 \\
\hline 1. Perceived stress & 1.000 & & & \\
\hline 2. Negative-focused cognitive emotion regulation & $0.629^{* * * *}$ & 1.000 & & \\
\hline 3. Reward sensitivity & $0.176^{* * * *}$ & $0.326^{* * *}$ & 1.000 & \\
\hline 4. Emotional eating & $0.282^{* * * *}$ & $0.262^{* * * *}$ & $0.194^{* * * *}$ & 1.000 \\
\hline
\end{tabular}

Note. $* \mathrm{p}<0.05 ; * * \mathrm{p}<0.01 ; * * * \mathrm{p}<0.001$ 
252

253

254

255

256

257

258

259

260

261

262

263

264

Mediation of the Relationship Between Perceived Stress and Emotional Eating

As shown in Fig. 1 , the total effect $(\beta=0.2557, p=0.0000,95 \% \mathrm{CI}=[0.1589$, 0.3526]) from perceived stress to emotional eating was at a significant level (Step 1). Moreover, the direct paths from perceived stress to negative-focused cognitive emotion regulation was significant $(\beta=0.6545, \mathrm{p}=0.0000,95 \% \mathrm{CI}=[0.5809$, 0.7281]). However, the direct paths from perceived stress to reward sensitivity was insignificant $(\beta=-0.0470, p=0.3824,95 \% \mathrm{CI}=[-0.1527,0.0586])$. Meanwhile, the paths from the first mediator (negative-focused cognitive emotion regulation) to the second mediator (reward sensitivity) were also significant $(\beta=0.3484, p=0.0000,95 \%$

$\mathrm{CI}=[0.2308,0.4660])($ Step 2). The paths from the mediators, namely, negative-focused cognitive emotion regulation $(\beta=0.1141, \mathrm{p}=0.0456,95 \% \mathrm{CI}=$ $[0.0022,0.2260])$ and reward sensitivity $(\beta=0.1353, \mathrm{p}=0.0061,95 \% \mathrm{CI}=[0.0388$, 0.2318]), to emotional eating were significant (Step 3). The direct path from perceived stress to emotional eating was significant $(\beta=0.1566, \mathrm{p}=0.0114,95 \% \mathrm{CI}$ $=[0.0355,0.2777])($ Step 4). Moreover, the mediating variables (negative-focused cognitive emotion regulation and reward sensitivity) were observed to exert a mediating effect on the relationship between perceived stress and emotional eating. 


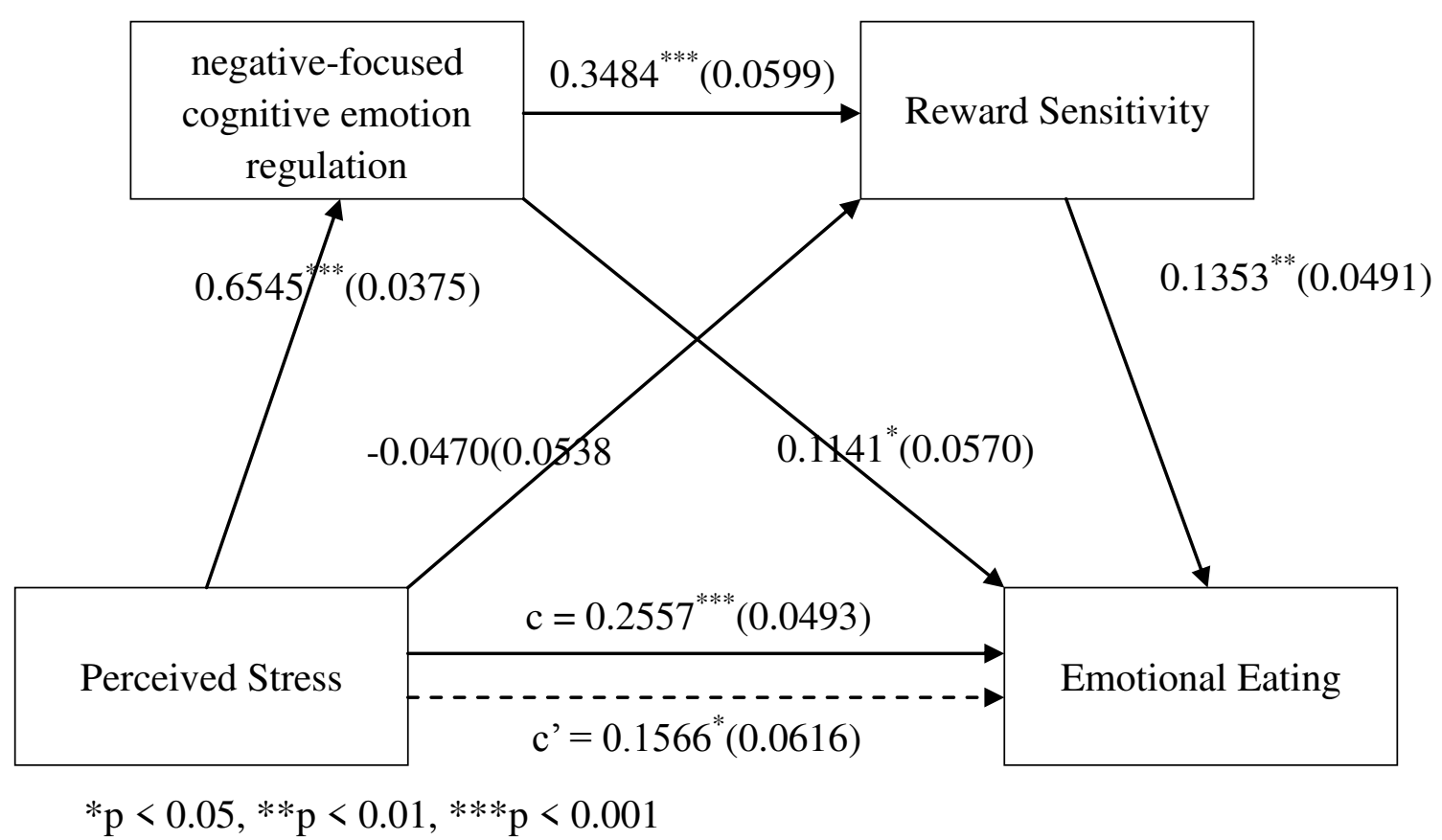

Fig. 1 The serial multiple mediation model of the present study

Table 3 shows the direct and indirect path estimates and bias-corrected CIs while controlling for sex, age, household income, and BMI. As shown in the table, the total indirect effect (i.e., the difference between total effect and direct effect) of perceived stress through negative-focused cognitive emotion regulation and reward sensitivity on emotional eating was significant $(b=0.0992,95 \% \mathrm{CI}=[0.0303,0.1738])$. The single mediation of negative-focused cognitive emotion regulation, and the multiple serial mediations of negative-focused cognitive emotion regulation and reward sensitivity were significant in the tested model, but the single mediation of reward sensitivity in the relationship between perceived stress and emotional eating was insignificant in the tested model.

Table 3 Bootstrapping indirect effects and $95 \%$ confidence intervals (CI) for the final mediational model 


\begin{tabular}{|c|c|c|c|c|}
\hline \multirow[t]{2}{*}{ Number } & \multirow[t]{2}{*}{ Model pathways } & \multirow[t]{2}{*}{ Point estimates $\beta$} & \multicolumn{2}{|l|}{$95 \% \mathrm{CI}$} \\
\hline & & & Lower & Upper \\
\hline 1 & Total Indirect Effect & 0.0992 & 0.0303 & 0.1738 \\
\hline 2 & $\mathrm{PS} \rightarrow \mathrm{NFCER} \rightarrow \mathrm{EE}$ & 0.0747 & 0.0025 & 0.1495 \\
\hline 3 & $\mathrm{PS} \rightarrow \mathrm{NFCER} \rightarrow \mathrm{RS} \rightarrow \mathrm{EE}$ & 0.0309 & 0.0099 & 0.0605 \\
\hline 4 & $\mathrm{PS} \rightarrow \mathrm{RS} \rightarrow \mathrm{EE}$ & -0.0064 & -0.0272 & 0.0063 \\
\hline
\end{tabular}

\section{Discussion}

This study aimed to examine the relationships between perceived stress and emotional eating among adolescents. Results indicated that for adolescents, increased perceived stress was directly associated with increased emotional eating via a pathway from higher negative-focused cognitive emotion regulation to higher reward sensitivity.

291 In line with prior studies, adolescents of higher perceived stress reported more

292 emotional eating behaviours [2, 8, 9], and perceived stress had a significant direct 293 effect and a significant total effect on emotional eating. People who live with chronic stress often cope with stressors by engaging in unhealthy behaviours, such as smoking, drug and alcohol abuse, and overeating. Because, eating more comfort food reduces

296 anxiety by reduce activity in the hypothalamo-pituitary-adrenal axis [45]. People who

297 exhibit emotional eating, derived from Kaplan's psychosomatic theory of obesity [27],

298 tend to overeat in order to reduce discomfort. Emotional eating may be learned [46].

299 In the classical conditioning framework, food intake is the unconditioned stimulus,

300 which is paired with unconditioned responses such as salivation and a desire to eat.

301 Over time, if perceived stress is systematically paired with food intake, it may become 302 a conditioned stimulus that can produce conditioned responses such as an increased 
desire to eat. In the operant-conditioning framework, successfully reducing negative affect through food intake is considered to reinforce emotional eating. Over time, if perceived stress is systematically paired with food intake, people may become conditioned to experience an increased desire to eat. Thus, the more stress a person perceived, the more emotional eating behaviours.

Serial multiple mediation analyses indicated that emotional eating may occur based on indirect associations made between perceived stress and emotional eating, not only a direct consequence of perceived stress. There was a significant indirect effect between perceived stress and emotional eating behaviour that was mediated by negative-focused cognitive emotion regulation. One theory is that bingeing occurs as part of an attempt to escape from negative self-awareness. Attention is focused on the immediate stimulus environment and away from more meaningful levels of cognition, allowing the individual to avoid dealing with ego-threatening information [19]. This narrowing of attention disengages normal inhibitions against eating and fosters an uncritical acceptance of irrational beliefs and thoughts. As maladaptive strategies fail to diminish the negative effect from stress, the adolescent may consequently turn to food as comfort. Thus, during stressful situations, the more negative-focused cognitive emotion regulation an adolescent had, the more emotional eating behaviours he or she had.

There was no significant indirect effect between perceived stress and emotional eating behaviour that was mediated by reward sensitivity, while reward sensitivity had a significant direct effect on emotional eating. Previous research found that stress 
would increase or diminish reward sensitivity [34]. However, we didn't find that stress had a significant direct effect on reward sensitivity. It may be due to individual differences of reward sensitivity influenced by genetic and environmental factors [47]; no significant effect was found in the limited sample size. It also suggested that some factors mediated the effect of stress on reward sensitivity, such as cognitive emotion regulation.

Furthermore, this study found that negative-focused cognitive emotion regulation and reward sensitivity significantly partially mediated the effect of perceived stress on emotional eating. The results indicated that adolescents had an increased tendency to approach rewarding stimuli such as highly palatable foods and then had more emotional eating behaviours, when they had more difficulties in coping with perceived stress. According to resource depletion theory [48], self's capacity for active volition is limited, all acts of self-control rely on the same limited resource. As such, the use of these resources for an initial act of self-control would reduce the resources available for a subsequent self-control behaviour. According to the theory of stress induced eating [27], the cognitive load of coping with a stressor may overwhelm self-regulatory resources so that people focus on eating rather than focusing on the stressor. Negative-focused cognitive emotion regulation consumed more resources; individuals thus failed to control their cravings for reward stimuli and eating behaviour properly after negative-focused cognitive emotion regulation. If cognitive emotion regulation fails, self-regulation in other areas, like control over eating behaviours, can fail as well [49]. Our study suggested that reducing negative-focused 
cognitive emotion regulation is necessary for the regulation of emotional eating. Therefore, the emotional eating of adolescents can be reduced by reducing maladaptive strategies. For example, cognitive emotion regulation could be improved by cognitive behaviour therapy and mindfulness-based therapy.

This study also showed that girls perceived more stress, used more negative-focused cognitive emotion regulation, and reported more emotional eating than boys. This could be because girls are under more pressure from school performance, relationships and looks than boys. In this study, age was negatively related to perceived stress and emotional eating. Younger adolescents just come to a new junior high school and face challenges in academic and extracurricular areas, and they need some time to adjusted to the new environment, thus they perceived more stress. Consistent with previous studies, age was positively related to reward sensitivity, because reward sensitivity increases from childhood to adulthood [29, 30].

It is also important to note several limitations. First, all participants come from one city; thus, the rates of perceived stress and emotional eating reported here should not be considered representative of different subgroups. To maximize the generalizability of future studies, randomized sampling should be performed at many sites. Furthermore, the measures utilized here all rely on self-report and may be subject to response bias. To reduce the impact of subjectivity, multiple methods should be developed and used for assessment [50]. However, self-report measures are commonly used to assess stress, cognitive emotion regulation, reward sensitivity, and eating behaviour, and the validity of the study instruments has been well established. 
Finally, no causal conclusions can be drawn because of the cross-sectional nature of the study. Nevertheless, these findings offer a first assessment of the mediating roles

371 of reward sensitivity and negative-focused cognitive emotion regulation in the 372 relationship between perceived stress and emotional eating in adolescence, utilizing 373 validated measures of study variables. Findings suggest a need for further research in 374 this area. Longitudinal studies should be undertaken to better assess whether reducing 375 negative-focused cognitive emotion regulation has a preventative or ameliorative role 376 in emotional eating and which maladaptive or adaptive cognitive strategies affects the 377 relationship between perceived stress and emotional eating. Future work should also assess the influence of perceived stress on emotional eating in different demographics, such as people in late adolescence and early adulthood. As our results only explained part of the association between perceived stress and emotional eating, future research should focus on other factors such as parental behaviours, peer influence, and social media in relation to emotional eating.

\section{Conclusions}

In summary, this study extended insights into the complex interactions among the perceived stress, negative-focused cognitive emotion regulation, reward sensitivity,

387 and emotional eating of Chinese adolescents. The important path from perceived stress to negative-focused cognitive emotion regulation to reward sensitivity to emotional eating determines the internal mechanism between perceived stress and emotional eating. The results of the current study can provide valuable guidance in 
391

implementing psychological interventions to reduce emotional eating and improve eating behaviours of adolescents. Avoiding stress can be used as a preventive therapy to help such adolescents to reduce emotional eating. It can also be used as an active therapy to help them manipulate the impact of negative-focused cognitive emotion regulation and reward sensitivity, thereby reduce emotional eating.

\section{Acknowledgements}

The authors are grateful to all students, parents, and school principals who participated in this study.

\section{Authors' contributions}

Xia Xu and Jiaai Huang conceived and designed the study. Jiaai Huang, Yuanjie Wang and $\mathrm{Na}$ Ye collected and interpreted the data. Jiaai Huang wrote the first draft of the manuscript. Jiaai Huang and Xia Xu modified the manuscript. Yuanjie Wang and $\mathrm{Na}$ Ye reviewed the manuscript. All authors critically revised the manuscript and approved the final version.

\section{Funding}

This research was supported by the Science Fund for Hubei Superior Discipline Groups of Physical Education and Health Promotion, National Natural Science Foundation of China (Grant No. 81971661), and Natural Science Foundation of Hubei Province (Grant No. 2016CFA098). 
414 Availability of data and materials

415 The datasets generated during and analysed during the current study are available in

416 the [Open Science Framework] repository, [https://osf.io/r74yh].

417

418 Ethics approval and consent to participate

419 The research protocol was approved by the Ethics Committee of Wuhan Sports 420 University.

Consent to publication

423

Not applicable.

424

425

Competing interests

426

The authors declare that they have no competing interests.

427

\section{References}

429

1. van Strien T, Oosterveld P. The children's DEBQ for assessment of restrained,

430 emotional, and external eating in 7- to 12-year-old children. International Journal of 431 Eating Disorders. 2008; 41(1): 72-81.

2. Vandewalle J, Moens E, Braet C. Comprehending emotional eating in obese youngsters: the role of parental rejection and emotion regulation. International Journal of Obesity. 2014; 38(4): 525-30. 
3. Debeuf T, Verbeken S, Van Beveren ML, Michels N, Braet C. Stress and Eating Behavior: A Daily Diary Study in Youngsters. Frontiers in Psychology. 2018; 9: 2657. 4. Stice E, Presnell K, Spangler D. Risk factors for binge eating onset in adolescent girls: a 2-year prospective investigation. Health Psychology. 2002; 21(2): 131-8.

5. Tsenkova V, Boylan JM, Ryff C. Stress eating and health. Findings from MIDUS, a national study of US adults. Appetite. 2013; 69: 151-5.

6. Erschens R, Herrmann-Werner A, Keifenheim KE, Loda T, Bugaj TJ, Nikendei C, et al. Differential determination of perceived stress in medical students and high-school graduates due to private and training-related stressors. PLoS One. 2018; 13(1): e0191831.

7. Hill DC, Moss RH, Sykes-Muskett B, Conner M, O'Connor DB. Stress and eating behaviors in children and adolescents: Systematic review and meta-analysis. Appetite. 2018; 123: 14-22.

8. Michels N, Sioen I, Braet C, Eiben G, Hebestreit A, Huybrechts I, et al. Stress, emotional eating behaviour and dietary patterns in children. Appetite. 2012; 59(3): 762-9.

9. Nguyen-Rodriguez ST, Unger JB, Spruijt-Metz D. Psychological determinants of emotional eating in adolescence. Eating Disorders. 2009; 17(3): 211-24.

10. Vandewalle J, Mabbe E, Debeuf T, Braet C, Moens E. The Daily Relation between Parental Rejection and Emotional Eating in Youngsters: A Diary Study. Frontiers in Psychology. 2017; 8: 691.

11. Gibson EL. Emotional influences on food choice: sensory, physiological and 
psychological pathways. Physiology \& Behavior. 2006; 89(1): 53-61.

12. Gibson EL. The psychobiology of comfort eating: implications for neuropharmacological interventions. Behavioural Pharmacology. 2012; 23(5-6): $442-60$.

13. Sinha R, Jastreboff AM. Stress as a common risk factor for obesity and addiction. Biological Psychiatry. 2013; 73(9): 827-35.

14. Greeno CG, Wing RR. Stress-induced eating. Psychological Bulletin. 1994; 115(3): 444-64.

15. Torres SJ, Nowson CA. Relationship between stress, eating behavior, and obesity. Nutrition. 2007; 23(11-12): 887-94.

16. Raio CM, Orederu TA, Palazzolo L, Shurick AA, Phelps EA. Cognitive emotion regulation fails the stress test. Proceedings of the National Academy of Sciences of the United States of America. 2013; 110(37): 15139-44.

17. Aldao A, Nolen-Hoeksema S, Schweizer S. Emotion-regulation strategies across psychopathology: A meta-analytic review. Clinical Psychology Review. 2010; 30(2): 217-37.

18. Garnefski N, Kraaij V, Spinhoven P. Negative life events, cognitive emotion regulation and emotional problems. Personality and Individual Differences. 2001; 30(8): 1311-27.

19. Evers C, Marijn Stok F, de Ridder DT. Feeding your feelings: emotion regulation strategies and emotional eating. Personality and Social Psychology Bulletin. 2010; 36(6): 792-804. 
479

480

481

482

483

484

485

486

487

488

489

490

491

492

493

494

495

496

497

498

499

500

20. Shriver LH, Dollar JM, Lawless M, Calkins SD, Keane SP, Shanahan L, et al. Longitudinal Associations between Emotion Regulation and Adiposity in Late Adolescence: Indirect Effects through Eating Behaviors. Nutrients. 2019; 11(3).

21. Polivy J, Herman CP. Causes of eating disorders. Annual Review of Psychology. 2002; 53: 187-213.

22. Czaja J, Rief W, Hilbert A. Emotion regulation and binge eating in children. International Journal of Eating Disorders. 2009; 42(4): 356-62.

23. Harrist AW, Hubbs-Tait L, Topham GL, Shriver LH, Page MC. Emotion regulation is related to children's emotional and external eating. Journal of Developmental and Behavioral Pediatrics. 2013; 34(8): 557-65.

24. Kubiak T, Vogele C, Siering M, Schiel R, Weber H. Daily hassles and emotional eating in obese adolescents under restricted dietary conditions--the role of ruminative thinking. Appetite. 2008; 51(1): 206-9.

25. Vandewalle J, Moens E, Beyers W, Braet C. Can we link emotional eating with the emotion regulation skills of adolescents? Psychology \& Health. 2016; 31(7): 857-72.

26. Aparicio E, Canals J, Arija V, De Henauw S, Michels N. The role of emotion regulation in childhood obesity: implications for prevention and treatment. Nutrition research reviews. 2016; 29(1): 17-29.

27. Park M, Quinn L, Park C, Martyn-Nemeth P. Pathways of the relationships among eating behavior, stress, and coping in adults with type 2 diabetes: A cross-sectional study. Appetite. 2018; 131: 84-93. 
28. Vervoort L, Clauwaert A, Vandeweghe L, Vangeel J, Van Lippevelde W, Goossens L, et al. Factors influencing the reinforcing value of fruit and unhealthy snacks. European Journal of Nutrition. 2017; 56(8): 2589-98.

29. Shulman EP, Smith AR, Silva K, Icenogle G, Duell N, Chein J, et al. The dual systems model: Review, reappraisal, and reaffirmation. Developmental Cognitive Neuroscience. 2016; 17: 103-17.

30. Walker DM, Bell MR, Flores C, Gulley JM, Willing J, Paul MJ. Adolescence and Reward: Making Sense of Neural and Behavioral Changes Amid the Chaos. The Journal of Neuroscience. 2017; 37(45): 10855-66.

31. De Cock N, Van Lippevelde W, Vervoort L, Vangeel J, Maes L, Eggermont S, et al. Sensitivity to reward is associated with snack and sugar-sweetened beverage consumption in adolescents. European Journal of Nutrition. 2016; 55(4): 1623-32.

32. Tomiyama AJ. Stress and Obesity. Annual Review of Psychology. 2019; 70: 703-18.

33. Berghorst LH, Bogdan R, Frank MJ, Pizzagalli DA. Acute stress selectively reduces reward sensitivity. Frontiers in Human Neuroscience. 2013; 7: 133.

34. Ironside M, Kumar P, Kang MS, Pizzagalli DA. Brain mechanisms mediating effects of stress on reward sensitivity. Current Opinion in Behavioral Sciences. 2018; 22: 106-13.

35. Davis C, Patte K, Levitan R, Reid C, Tweed S, Curtis C. From motivation to behaviour: a model of reward sensitivity, overeating, and food preferences in the risk profile for obesity. Appetite. 2007; 48(1): 12-9. 
36. Monteleone P, Scognamiglio P, Monteleone AM, Perillo D, Maj M. Cortisol awakening response in patients with anorexia nervosa or bulimia nervosa: relationships to sensitivity to reward and sensitivity to punishment. Psychological Medicine. 2014; 44(12): 2653-60.

37. Hasking PA. Reinforcement sensitivity, coping, disordered eating and drinking behaviour in adolescents. Personality and individual differences. 2006; 40(4): 677-88. 38. Hundt NE, Williams AM, Mendelson J, Nelson-Gray RO. Coping mediates relationships between reinforcement sensitivity and symptoms of psychopathology. Personality and Individual Differences. 2013; 54(6): 726-31.

39. Ivory NJ, Kambouropoulos N. Coping mediates the relationship between revised reinforcement sensitivity and alcohol use. Personality and Individual Differences. 2012; 52(7): 822-7.

40. Tull MT, Gratz KL, Latzman RD, Kimbrel NA, Lejuez CW. Reinforcement sensitivity theory and emotion regulation difficulties: A multimodal investigation. Personality and Individual Differences. 2010; 49(8): 989-94.

41. Chen YL, Kuo PH. Effects of perceived stress and resilience on suicidal behaviors in early adolescents. European Child and Adolescent Psychiatry. 2019.

42. Hayes AF. Introduction to mediation, moderation, and conditional process analysis: A regression-based approach. In: Introduction to mediation, moderation, and conditional process analysis: A regression-based approach: xvii, 507-xvii, . Guilford Press, 2013.

43. Preacher KJ, Hayes AF. Asymptotic and resampling strategies for assessing and 
comparing indirect effects in multiple mediator models. Behavior Research Methods. 2008; 40(3): 879-91.

44. Podsakoff PM, MacKenzie SB, Lee JY, Podsakoff NP. Common method biases in behavioral research: a critical review of the literature and recommended remedies. Journal of Applied Psychology. 2003; 88(5): 879-903.

45. Dallman MF, Pecoraro N, Akana SF, La Fleur SE, Gomez F, Houshyar H, et al. Chronic stress and obesity: a new view of "comfort food". Proceedings of the National Academy of Sciences of the United States of America. 2003; 100(20): 11696-701.

46. Altheimer G, Urry HL. Do Emotions Cause Eating? The Role of Previous Experiences and Social Context in Emotional Eating. Current Directions in Psychological Science. 2019; 28(3): 234-40.

47. Richards JS, Arias Vasquez A, von Rhein D, van der Meer D, Franke B, Hoekstra PJ, et al. Adolescent behavioral and neural reward sensitivity: a test of the differential susceptibility theory. Translational Psychiatry. 2016; 6: e771.

48. Baumeister RF, Bratslavsky E, Muraven M, Tice DM. Ego depletion: is the active self a limited resource? Journal of Personality and Social Psychology. 1998; 74(5): $1252-65$.

49. Leehr EJ, Krohmer K, Schag K, Dresler T, Zipfel S, Giel KE. Emotion regulation model in binge eating disorder and obesity - A systematic review. Neuroscience and Biobehavioral Reviews. 2015; 49: 125-34.

50. Braden A, Emley E, Watford T, Anderson L, Musher-Eizenman D. Self-reported 
567 emotional eating is not related to greater food intake: results from two laboratory 568 studies. Psychology \& Health. 2020; 35(4): 500-17.

569

570 
Figures

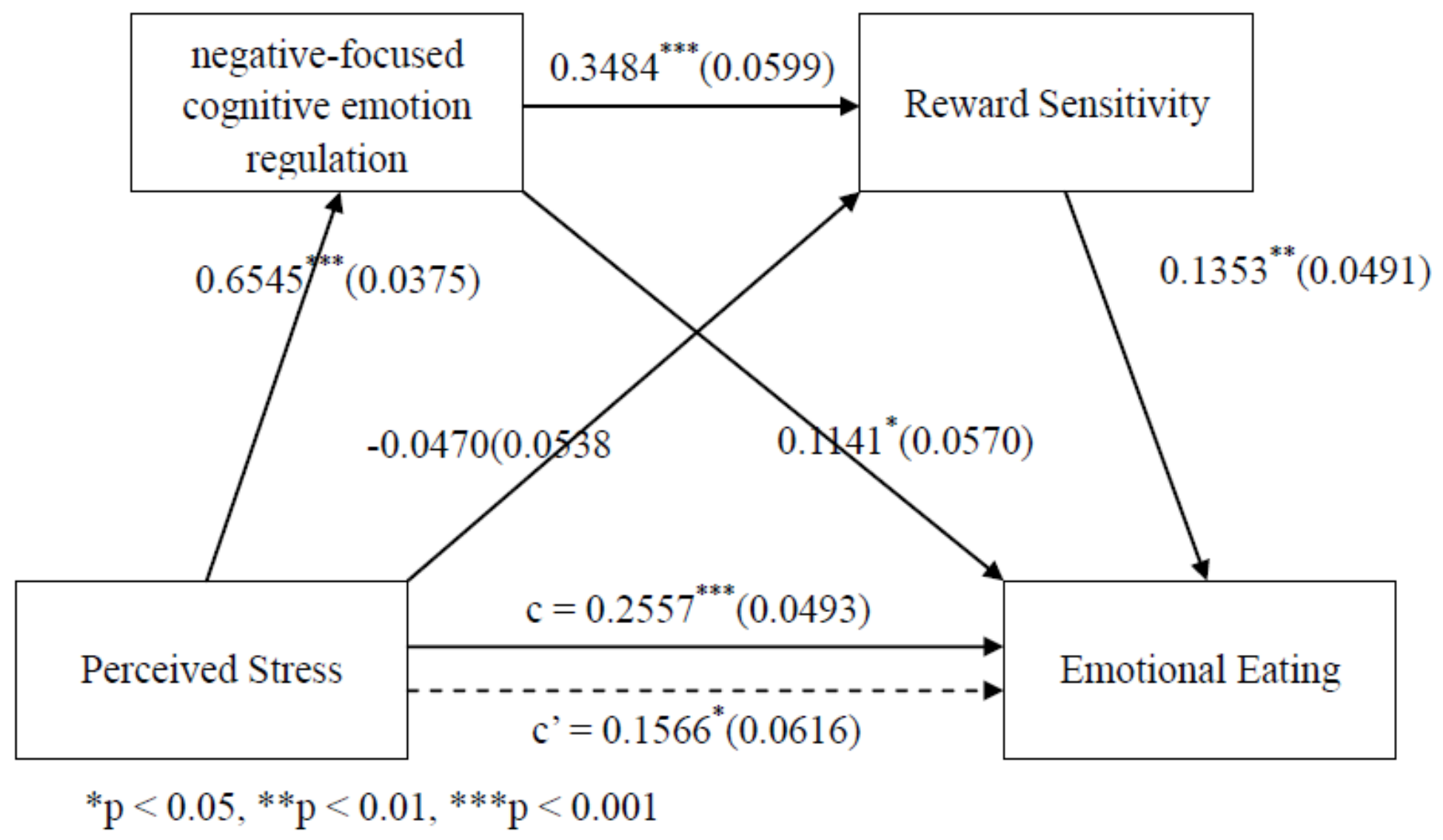

Figure 1

The serial multiple mediation model of the present study 